\title{
28 \\ COLOUR CATEGORIZATION AND CATEGORICAL PERCEPTION
}

\author{
Robert Briscoe
}

\section{Introduction}

Human beings with normal, trichromatic vision have the capacity to discriminate approximately 2 million different shades of colour (Pointer and Attridge 1998; Linhares et al. 2008; Kuehni 2013). ${ }^{1}$ Despite the fine-grained specificity with which we perceive colour, we tend to think and speak about colour in terms of a comparatively much small number of coarse-grained categories. English, for example, contains only 11 basic colour terms (BCTs)—'black', 'white', 'red', 'yellow', 'green', 'blue', 'brown', 'orange', 'pink', 'purple', and 'grey'-while the unwritten languages of many non-industrialized societies contain as few as two or three BCTs. ${ }^{2}$

A long-standing debate in cognitive science, linguistics, anthropology, and philosophy concerns the basis of human colour categorization and naming practices. Are there psychologically or culturally universal constraints on how speakers of different languages sort fine-grained shades of colour into compact sets of coarse-grained categories? Or is it rather the case that 'each culture has taken the spectral continuum and divided it on a basis which is quite arbitrary except for pragmatic considerations' (Ray 1953: 102)? One aim of this chapter is to survey some of the recently more influential ways of answering these questions.

Another related question concerns the relationship between colour naming practices and colour perception. Do linguistic representations of colour categories influence the way human beings visually experience colour? In particular, can learning to use a set of colour terms cause shades that straddle a category boundary to appear phenomenally less similar (and, hence, easier to discriminate) or cause shades that fall within the boundaries of a named category to appear phenomenally more similar (and, hence, harder to discriminate)? If so, then visually perceiving colour, like phoneme discrimination in language (Liberman and Mattingly 1985; Harnad 1987; Kuhl 2004), may be described as 'categorical'. Alternatively, linguistic representations of colour categories might influence the speed or accuracy with which fine-grained shades colours are discriminated or remembered without having any effects on their appearance.

The colour categorization debate has been traditionally framed as a conflict between 'universalist' and 'relativist' conceptions of the relation between language, thought, and perception (for helpful overviews, see Dedrick 1998, 2014a; Hardin 2005; Jameson 2005b; Regier and Kay 2009; Roberson and Hanley 2009; Regier et al. 2010; Roberson 2012; Winawer and Witthoft 
2012; and Lindsey and Brown 2014). ${ }^{3}$ To simplify greatly, strong universalism maintains (1) that coarse-grained colour concepts corresponding to BCTs are unlearned, psychological universals that recur across maximally different cultures; (2) that the psychological universality of these colour concepts is due to the perceptual salience of their best examples or 'foci'; and, finally, (3) that the representation of colour categories in language has no influence on the way colours are represented at the level of either thought or perception. Strong linguistic relativism, in contrast, denies claim (1): basic colour concepts aren't psychological universals, but vary instead with cultural and communicative needs. Hence, there are no cross-cultural patterns in colour categorization and colour-naming practices that demand scientific explanation, as assumed by claim (2). Strong linguistic relativists also deny claim (3), maintaining instead that colour terms are the primary vehicles of colour category representation and, strikingly, that an object's apparent colour can vary as a function of the colour terms present in the perceiver's language. In denying claim (3), strong linguistic relativism has an affinity with the writings of Benjamin Lee Whorf (1956).

As will be shown below, this stark way of framing the colour categorization debate has probably outlived its usefulness. On the one hand, prominent universalists no longer maintain that considerations of perceptual salience are what best account for the cross-cultural recurrence of certain basic colour concepts. They also accept that colour language can have experimentally measurable effects on colour memory and colour discrimination tasks. In other words, they have distanced themselves from claims (2) and (3) above. On the other hand, prominent linguistic relativists now deny that so-called colour 'categorical perception' effects are properly interpreted as effects of language on the way colours visually appear. Speakers of different languages do not experience the colours present in their environment in different ways. Both sides of the debate, in short, have moved closer toward the moderate centre of the theoretical spectrum between strong universalism and strong linguistic relativism.

In the next two sections of this chapter, I critically examine two of the main approaches to colour categorization in cognitive science: the perceptual salience theory and linguistic relativism. I then turn to reviewing several decades of psychological research on colour categorical perception (CP). A careful assessment of relevant findings suggests that most of the experimental effects that have been understood in terms of $\mathrm{CP}$ actually fall on the cognition side of the perceptioncognition divide: they are effects of colour language, for example, on memory or decisionmaking.

\section{The perceptual salience theory}

Theories of colour categorization can be distinguished by the constraints that they respectively impose on the colour concept formation process. Two constraints, however, appear to be common ground across different theories. The first is grouping by similarity (or grouping for short). Debi Roberson and co-authors write:

There are, indeed, constraints on color categorization linked to the properties of the visual system. The most important constraint would be that similar items (as defined by perceptual discrimination) are universally grouped together. Thus, no language would exhibit categories that include two areas of color space but excludes an area between them. ... Grouping by similarity can explain, for example, why there is no composite category that includes yellow and blue but excludes green. There is no associative chain of similarity that could connect yellow to blue without passing through green.

(2000: 395) 
A second shared constraint is that colour categorization systems are constructed so as maximize perceptual similarity within categories, while minimizing perceptual similarity between different categories (Garner 1974). Since systems that comport with this constraint, other things being equal, are more informative, i.e. communicatively efficient, than those that don't (Jameson and D’Andrade 1997; Jameson 2005a, 2005b; Regier et al. 2007; Regier et al. 2015), I shall here refer to it as the informativeness constraint.

Proponents of the perceptual salience theory (Rosch 1973; Kay and McDaniel 1978; Hardin 1988, 2005; Kay et al. 1997; Kay and Maffi 1999; Kuehni 2005a; Kay and Regier 2007) maintain that a third universal constraint on colour categorization and naming comes from the way in which human beings experience colour. The 'basic linguistic categories themselves', C.L Hardin writes, 'have been induced by perceptual saliencies common to the human race' (1988: 168).

According to the theory, certain shades of colour, in particular, the 'Hering primaries' black, white, unique red (UR), unique yellow (UYY), unique green (UG), and unique blue (UBB) (Hering 1878/1964), are especially salient in visual experience prior to their representation in either language or thought. (I shall follow Byrne and Hilbert 2003 in referring to these chromatic shades collectively as the $\mathbf{4} \mathbf{U H}$.) The perceptual salience or 'attention-grabbingness' of these shades is often said to arise from their distinctively pure or non-mixed appearance. As Justin Broackes puts it, 'There are no purples that do not look to have some red and some blue in them, no turquoises that do not in some way seem bluish and also greenish; but there are reds that don't look in any way bluish or yellowish and yellows that seem to contain no hint of either red or green' (Broackes 2011: 602). It has also been argued that terms for the $4 \mathrm{UH}$ are both necessary and sufficient for linguistically describing all of the other colours (Sternheim and Boynton 1966; Hardin 1981, chap. 5). This fact, Hardin says, 'justifies singling them out as perceptually elementary' and as having 'psychological primacy' (2005: 74). Byrne and Hilbert (2003) go beyond Hardin in arguing that colours are actually represented in visual experience itself in terms of the proportions of the primary hue-magnitudes that they contain. For example, a surface will look purple to a perceiver just in case it is represented in her experience as having roughly equal proportions of UR and UB, but relatively low proportions of UG and UY (Byrne and Hilbert 2003: 14). ${ }^{4}$

According to the best known version of the salience theory, the Hering primaries function as 'natural prototypes' in colour concept formation (Rosch 1973): whether a shade belongs in the category red, for example, is based on its perceived similarity to UR; whether a shade is in the category blue is based on its perceived similarity to UB; and so on. The Hering primaries, as Paul Kay and Luisa Maffi put it, thus function as 'perceptual landmarks [that] individually or in combination form the basis of the denotation most of the major color terms of most of the languages of the world' (1999: 774).

The perceptual salience theory is an expression of universalism about the relationship between language and thought in the colour domain. '[F]ar from being a domain well suited to the study of the effects of language on thought', Eleanor Rosch concluded at the end of her influential 'Universals in color naming and memory' (Rosch Heider 1972), 'the color space would seem to be a prime example of the influence of underlying perceptual-cognitive factors on the formation and reference of linguistic categories' (20). Unlike the relativist views considered below, universalism maintains that, in representing colour, 'languages make semantic distinctions drawn from a palette of universally available options' (Regier et al. 2010: 165).

Early sources of empirical support for the salience theory came from Rosch's pioneering studies of colour naming and colour memory among the Dugum Dani, a hunter-gatherer tribe living in the highlands of western New Guinea (Rosch Heider 1972; Rosch Heider and Olivier 1972; 
Rosch 1973). One of Rosch's goals was to test the linguistic relativistic hypothesis that 'verbal color coding acts on memory imagery such that the "structure" of colors in memory comes to resemble the "structure" of color names in a given language' (Rosch Heider and Olivier 1972: 338). The Dani have only two BCTs, which divide colour space into warm-light and cool-dark regions. Hence, linguistic relativism predicts that the Dani performance on colour memory tasks should significantly differ from that of English speakers, who use many more BCTs.

At odds with what the linguistic relativist would predict, Rosch and Olivier found 'no indication that the differences between the naming structures for the two languages carried over in parallel fashion to the two memory structures' (1972: 350). On the contrary, the Dani and English speakers exhibited similar confusions in memory. For instance, good examples of colour categories named in English such as red, blue, and green were better remembered by Dani participants than colours less easily named by English speakers. These findings were taken to furnish strong support for the view that certain perceptually salient colours are 'the cognitive underpinning for cross-language naming universals' (Kay and Regier 2007: 290).

This account was subsequently challenged two decades later by the work of Jules Davidoff, Debi Roberson, and others on the Berinmo of Papua New Guinea and the Himba of Northern Namibia (Davidoff et al. 1999; Roberson et al. 2000; Roberson et al. 2002; Roberson et al. 2005a). Davidoff and Roberson found no evidence in their studies of short-term memory and long-term category learning indicating that Berinmo and Himba speakers find it easier to recall basic colour categories named in English than those named in their own languages. Further, in similarity and forced-choice recognition memory judgement tasks, Berinmo and Himba speakers exhibited categorical perception (CP) effects for colour boundaries that were marked in their own languages, but not for the supposedly universal boundary between green and blue marked in English and other written languages (Roberson et al. 2005b). (These studies and the proper interpretation of putative colour CP effects will be addressed later in this chapter.)

A second, more important source of evidence for the salience theory is linguistic. To date there have been two large-scale investigations of cross-cultural colour-naming practices, the landmark study of 20 different written languages conducted by Brent Berlin and Paul Kay (1969) and the more recent World Color Survey (WCS), which identified BCTs in an additional 110 unwritten languages (Kay et al. 2009). On the basis of their findings, Berlin and Kay advanced two hypotheses. First, 'although different languages encode in their vocabularies different numbers of basic color categories, a total inventory of exactly eleven basic color categories exists from which the eleven or fewer basic color categories of any given language are always drawn' (Berlin and Kay 1969: 2). In the lexicon of American and British English, the 11 basic categories are picked out by the terms 'black', 'white', 'red', 'yellow', 'green', 'blue', 'brown', 'orange', 'pink', 'purple', and 'grey'. Second, BCT inventories evolve through time by incorporating these categories in a highly constrained sequence, starting with just two BCTs referring to the composite categories warm/light and dark/cool.

The World Color Survey (WCS) collected colour naming data from 110 unwritten languages spoken in small, non-industrialized societies (Kay et al. 2009). Speakers were asked to name each of 320 maximally saturated Munsell colours and 10 grey-scale colours, presented in random order, from the array reproduced in Figure 28.1(A). They were also asked to demonstrate the best examples or foci of each of their named colours.

Regier et al. (2005) calculated how many best-example 'hits' fell on each chip of the array for all speakers interviewed in the WCS. The contour plot in Figure 28.1(B) shows the number of WCS best-example hits that fell on each chip in the chromatic portion of the stimulus array (with a contour interval of 100 hits). The black dots correspond to the foci of the English colour terms 'red', 'yellow', 'green', and 'blue' provided by one American speaker (Berlin and Kay 
(A)

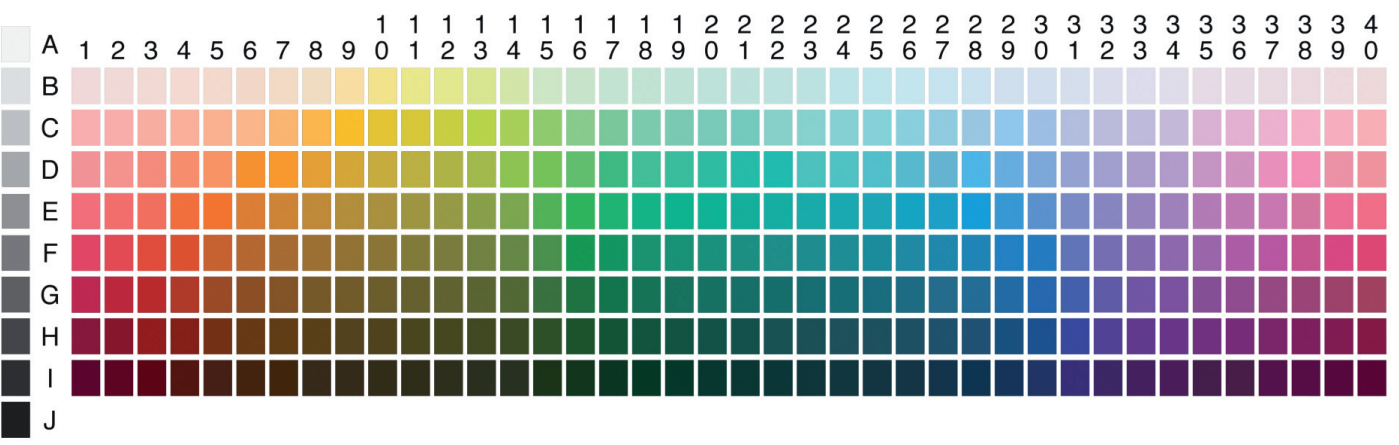

(B)

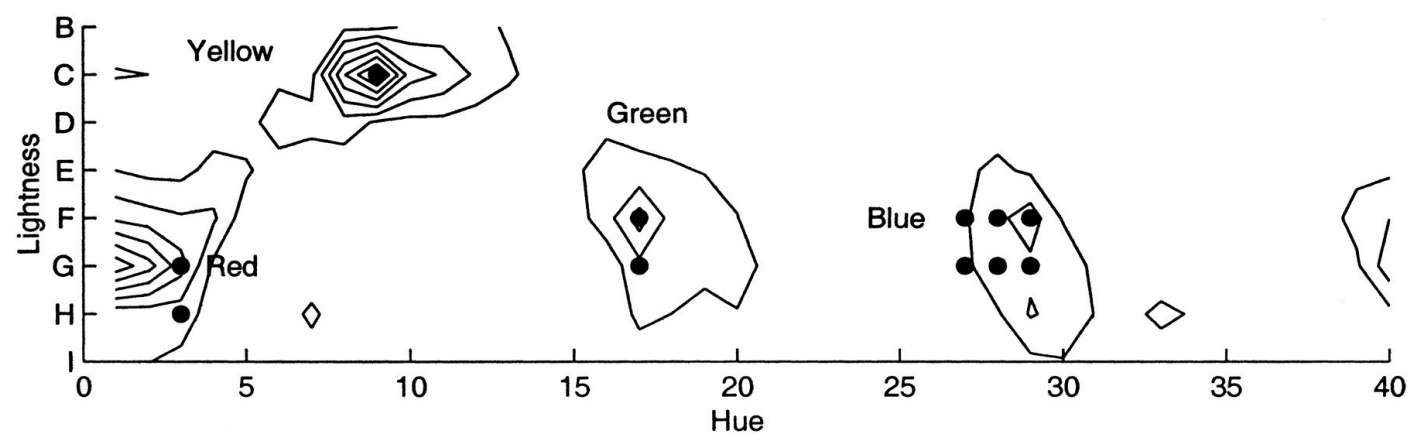

Figure 28.1 (A) Munsell colour palette and (B) contour plot of WCS best-example choices compared with best examples of English colour terms for the chromatic Hering primaries.

1969). As indicated by the contour plot, the best examples of named chromatic colour categories across the 110 languages of the WCS cluster around the best examples of four English terms: G1 (focal red), C9 (focal yellow), F17 (focal green), and F29 (focal blue). Regier et al. (2005) also found that best examples of BCTs are more tightly clustered across the languages of the WCS than are the centroids of category extensions. 'This pattern', they write,

would be expected if best examples reflect universal foci against a background of crosslinguistically varying category extensions. However, it would not be predicted if best examples are abstracted instead as the centers of categories defined at their boundaries by linguistic convention, because on this latter view, best examples are category centers and will cluster only as tightly as those centers.

(2005: 8389)

Critics have posed a number of challenges to the methodology used to collect cross-cultural naming data for the WCS. Use of highly saturated colour stimuli, Roberson and Hanley (2009) argue, may have led researchers to overestimate the similarity of colour categorization systems across different languages. Others have questioned the foundational assumption that every language contains a set of BCTs in the sense of Berlin and Kay (1969) (Levinson 1997).

These methodological criticisms notwithstanding, there appears to be converging linguistic evidence for the existence of cross-cultural tendencies in colour-naming practices. Lindsey and 
Brown (2006) performed a cluster analysis on the individual colour-naming systems of the 2,367 informants in the WCS. At the level of eight clusters, they found a close correspondence between clusters in the WCS naming data and familiar English chromatic colour categories. Two exceptions were a composite yellow-or-orange category, and, a composite green-or-blue category (grue). A second cluster analysis by Lindsey and Brown (2009) found that WCS colournaming systems can be divided into approximately four recurrent patterns or 'motifs'.

Despite evidence for the existence of cross-cultural tendencies in colour naming-practices, the perceptual salience theory has been recently abandoned as an account of how lexicalized colour categories are formed. Three main objections recur in the literature. First, even if the best examples of colour categories across many languages in the WCS cluster around the Hering primaries (4UH plus black and white), the perceptual salience theory is only a name for this fact, not explanation of it (Byrne and Hilbert 1997; Dedrick 1997; Jameson 2005a, 2005b). Regier and co-authors (2005) appear to concede this point:

The degree to which ... universally favored regions [of color space] are based on color appearance, universal statistical tendencies in the distribution of reflective surfaces in the environment, universal properties of ambient light sources, the topography of perceptual color space, or sociolinguistic negotiation among speakers cannot be assessed with any degree of certainty at this time. It is possible that all these factors, and perhaps others, play a role.

(Regier et al. 2005: 8390)

A second objection has to do with the variation in unique hue settings across observers with normal, trichromatic colour vision. Figure 28.2 presents the Munsell hue diagram with angular ranges in unique hue selections for approximately 300 normal, trichromat subjects (Kuehni $2005 \mathrm{~b})$. The broadness of the ranges of stimuli selected for the $4 \mathrm{UH}$ hues is large, with variation in settings for UG alone spanning nearly 30 per cent of the complete hue circle. One surprising consequence of this variability is that some observers will select as their best example of orange a stimulus that other observers respectively choose as their best example of UY or UR (Malkoc et al. 2005: 2156). Such dramatic variability in the way human beings perceive colour speaks

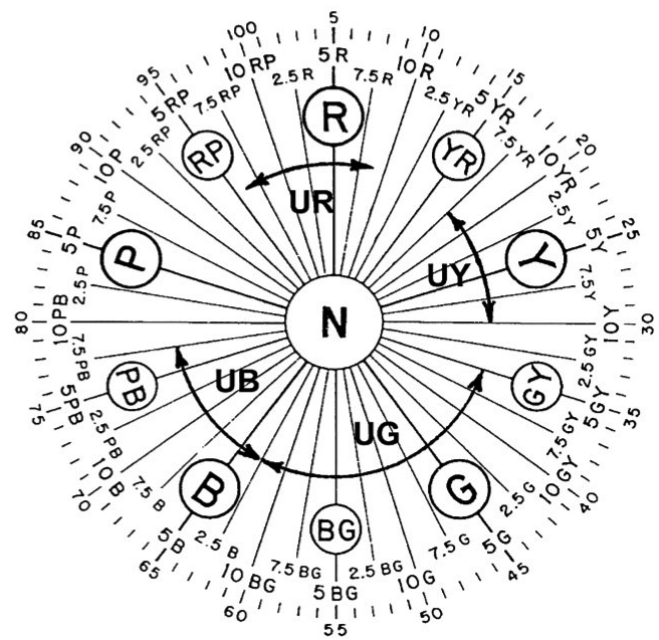

Figure 28.2 Munsell hue diagram with ranges of colour chip stimuli selected for the four unique hues. Source: reproduced with permission from Kuehni (2005a). 
against the view that the $4 \mathrm{UH}$ function as pan-human 'perceptual landmarks' that stabilize colour naming practices within and across linguistic communities (Jameson 2005b).

A final set of criticisms pose empirical objections to the assumption that the $4 \mathrm{UH}$ are perceptually more salient than other shades. Boynton (1997) found that when criteria of intrasubjective consistency in naming, consensus in naming, and short response times are applied, 'there are no differences between primary and derived basic colors [like pink and brown] except for the compound sensory aspect of the latter, which really does not seem to matter' (148). Similarly, Smallman and Boynton $(1990,1993)$ report that when embedded in visual information displays, best examples of English BCTs are not detected faster than other shades that are as widely separated in colour space. More recent studies have also failed to find greater intersubjective consistency in stimuli selection for the $4 \mathrm{UH}$ than for binary hues. Malkoc et al. (2002), in fact, report more consistency in subjects' choices for 'focal' blue-green than for UB and UG: in other words, there was less variability in selecting the boundary between blue and green than in selecting the best example of either category. Relatedly, Bosten and LawranceOwen (2014) found that subjects do not select examples of the 4UH in a display containing a complete hue circle more reliably than they select best examples of binary hues. According to Hardin, names for the $4 \mathrm{UH}$ are necessary and sufficient for naming all of the other colours, 'a fact that justifies singling them out as perceptually elementary' (2005: 74). A recent study by Bosten and Boehm (2014) challenges this assessment. Subjects were assigned to one of two experimental conditions in a hue-scaling experiment. In the 'unique' condition, they rated the proportions of UR, UY, UB, and UG that they perceived in each of a series of test stimuli. In the 'intermediate' condition, they rated the proportions of the binary colours teal, purple, orange, and lime. Results from the two conditions were found to be broadly the same. English speakers, Bosten and Boehm conclude, don't need to use names for the $4 \mathrm{UH}$ in order adequately to describe colour appearances.

In the last decade, erstwhile proponents of the perceptual salience theory have gravitated toward the view that cross-cultural patterns in colour naming may result from application of the informativeness constraint together with the irregular shape of colour space, as originally proposed by Kimberly Jameson and Roy D'Andrade (1997). Jameson and D'Andrade point out that the colour solid isn't a smooth globe, but an irregular blob with several large 'bumps'. For example, the regions around UR and UY achieve more saturation and, hence, protrude more from the solid than do the regions around UB and UG. The bumpy shape of perceptual colour space, they argue, means that certain ways of partitioning colour space into a small number of categories will be more informative than others (Jameson and D'Andrade 1997: 313; see also Jameson 2005a).

Building on this interpoint-distance model (IDM), Terry Regier and co-authors have recently proposed a 'shape-based' account of colour categorization, according to which naming systems across languages partition colour space in different, but close to optimally informative ways (Regier et al. 2007; Regier et al. 2015): 'The hypothesis is that ... irregularities in [colour] space, interacting with general principles of categorization, cause natural clusters to form that correspond to observed color-naming universals' (Regier et al. 2007: 1437). Regier et al. (2007) introduce the notion of well-formedness as a measure of the extent to which a lexical colour categorization system maximizes perceptual similarity of colours within a category and minimizes it across categories, where the perceptual similarity of two shades is inversely related to the distance between them in the CIELAB colour space. Colour-naming systems documented in the WCS, they argue, tend to have higher well-formedness than do systematic variants with the same number of categories, and attested divergences in the location of category boundaries tend to have only a minor impact on relative well-formedness. On this approach, cross-cultural 
patterns in colour naming practices aren't explained by any privileged set of focal colours. Instead, they result from the structure of perceptual colour space, the pragmatic need to communicate efficiently about colour, and cognitively universal categorization principles.

The IDM is perhaps the most prominent of recent attempts to explain recurrent patterns in colour-naming practices without appealing to the perceptual salience of the 4UH. Other approaches have also attracted attention. Yendrikhovskij (2001), building on Shepard (1992), links the structure of human colour categorization systems to the statistical distribution of colours in the natural environment. Steels and Belpaeme (2005) employ theoretical models and computer simulations of artificial agents to investigate the different ways in which the physiology of the human visual system, the colour statistics of natural scenes, and communicative needs respectively constrain the acquisition of a shared set of colour categories. Jameson and Komarova (2009a, 2009b) use agent-based, evolutionary game theory to explore the consequences of empirically observed heterogeneity in human colour-processing mechanisms, e.g. the absence of either long- or short-wavelength cones in dichromat observers, for the development colour categorization systems. They argue that evolved colour categorization systems tend to optimize communication among all members of the population, rather than only among members of the majority trichromat subset.

\section{Linguistic relativism}

Moderate linguistic relativism, as I shall call it, comprises three distinct claims:

(LR1) There are no psychologically universal constraints on colour categorization beyond perceptual grouping and informativeness and the structure of perceptual colour space (Roberson et al. 2000; Jameson 2005a, 2005b; Roberson et al. 2005b). Other non-universal constraints come from cultural or pragmatic needs, e.g. the need to distinguish in communication between edible and non-edible fruits, as well as from the distribution of shades in the natural and social environment.

(LR2) The process of colour category formation begins with boundary demarcation, and best examples or foci are extracted only at secondary stage of conceptual development (Roberson et al. 2000).

(LR3) Colour terms are the primary vehicles of colour category representation (Quine 1973; Roberson et al. 2000; Davidoff 2001; Roberson et al. 2005b): 'the results of recent experimental research would suggest that there are no cognitive color categories that are independent of the terms used to describe them' (Roberson 2005: 66). This claim reflects a robustly 'cognitive' conception of colour language. ${ }^{5}$

Strong linguistic relativism endorses a fourth, additional claim concerning the influence of colour terminology on the content and phenomenal character of colour experience.

(LR4) Learning to use a set of BCTs can cause shades that fall within the boundaries of a named category to appear phenomenally more similar to one another in appearance and shades that fall on opposite sides of a category boundary to appear phenomenally less alike. The 'structure of linguistic categories', as Davidoff puts it, 'distorts perception by stretching perceptual distances at category boundaries'.

(2001: 386) 
In maintaining LR1, linguistic relativists deny that certain perceptually salient shades constrain processes of colour concept formation (Roberson et al. 2000). The perceptual grouping and informativeness constraints by themselves, however, place only loose restrictions on the construction of colour categorization systems. Among other things, they leave open how the different, independently variable dimensions of colour appearance, that is, hue, saturation, and lightness, are to be respectively weighted in perceptual grouping, as attested by the significant amount of variability in weightings across empirically observed categorization systems (Jameson 2005a, 2005b). Further, application of the grouping constraint, as Dedrick has pointed out, presupposes prior identification of certain 'chromatic landmarks':

... there is no principled way to delimit the range of a linguistic color category that is constructed on the basis of generalization from a single sample. No way, that is, to know when to stop the process of associating color samples to one another. This problem is solved if judgement involves relative similarity: sample $x$ is more like $A$ than like $B$. With $A$ and $B$ (or whatever number of landmark colors) fixed, there is a cognitive constraint upon attribution of category membership.

(Dedrick 1998: 156)

The informativeness constraint also leaves a lot of wiggle room in the construction of colour categorization systems. It ensures that systems will be communicatively efficient, but it does not specify how many categories a system should contain.

On analogy with Chomsky's 'principles-and-parameters' approach to linguistic syntax (Chomsky 1995), we can think of grouping and informativeness as universal cognitive rules that govern the production of colour categorization systems. The selection of chromatic landmarks, dimensional weightings, and number of categories, in turn, can be thought of as setting parameters on application of these universal rules. It is a core tenet of linguistic relativism that the values of these parameters are determined locally by culture and language. This means that considerable variation across colour naming systems is, in principle, possible. ${ }^{6}$

According to the perceptual salience theory, basic colour concepts are formed by setting up boundaries around regions in three-dimensional colour space centred on the Hering primaries. In this respect, the representation of category foci or best examples is psychologically prior to the representation of category boundaries. Linguistic relativism, by contrast, maintains that the process of colour category formation begins with the demarcation of boundaries in colour space that are significant to observers for perhaps culturally quite local reasons (LR2).

An illustrative example is the wor-nol category boundary in Berinmo (Roberson et al. 2000). The term 'wor' applies to leaves that are ready to fall from a tree, covering shades of yellow, orange, khaki, and brown. The term 'nol' covers shades of chartreuse, green, blue, and purple This wor-nol boundary, Roberson and her co-authors emphasize, is far from arbitrary: 'tulip leaves, a favorite vegetable, are bright green when freshly picked and good to eat, but quickly yellow if kept. Agreement over the [wor-nol] boundary coincides with agreement over when they are no longer good to eat and is highly salient in a community that talks little about color' (Roberson et al. 2000: 395). By contrast, the ability to identify certain shades as the best examples or foci of the categories wor and nol is pragmatically much less important to the Berinmo and is argued to emerge only during a second phase of conceptual development: 'Once a category has been delineated at the boundaries, exposure to exemplars may lead to the abstraction of a central tendency so that observers behave as if their categories have prototypes' (Roberson et al. 2000: 395). 
The view that colour terms shape the way human beings think about colour is supported by empirical findings adduced on behalf of LR3, that is, the claim that colour terms are the primary vehicles of colour category representation. On this view, internalized colour language is the medium in which human beings think thoughts involving coarse-grained colour categories.

Support for LR3 comes from neuropsychological studies of subjects with colour naming deficits (but otherwise normal vision) who also exhibit impairments in the performance of seemingly non-linguistic colour categorization tasks. Roberson et al. (1999) report that a neuropsychological patient with severe impairments in colour naming is unable to sort coloured stimuli into groups except by pair-wise similarity. The same patient is also unable to judge which of three objects differs from the other two in an odd-colour-out task (Davidoff and Roberson 2004). Similar findings have been reported by the Lupyan lab at the University of Wisconsin. Lupyan and Mirman (2013) asked aphasic subjects to select objects from a group of 20 pictured objects using either a high-dimensional, 'thematic' category criterion (e.g. FRUIT, TOOLS, or FARM ANIMALS) or a low-dimensional, 'taxonomic' category criterion (e.g. BLUE, SMALL, or ROUND). They found that aphasics do not perform well on trials that require selection on the basis of a low-dimensional criterion and that the degree of impairment was predicted by their previously assessed naming performance. Categories 'held together by one or a small number of dimensions', Lupyan and Mirman write,

may require more on-line support from language. For example, the ability to selectively attend to objects having a particular color-classifying objects into a category of RED THINGS - may be facilitated by naming insofar as words such as 'red' help to group together objects that do not have pre-existing semantic associations and which differ substantially in surface appearance (e.g., a cherry and a brick).

(2013: 1191)

Consistent with this view, there is evidence that verbal interference selectively impairs normal subjects' ability to focus on particular perceptual dimensions such as size or colour. In fact, under verbal interference conditions, normal subjects have been reported to perform much like aphasic patients in odd-colour-out tasks (Lupyan 2009).

Additional support for LR3 come from studies of colour term acquisition and colour memory. Roberson and co-authors studied colour name learning and colour memory patterns in Berinmo and Himba speakers (Roberson et al. 2000; Roberson et al. 2005a; Roberson et al. 2005b). Contrary to findings garnered by Eleanor Rosch (Rosch Heider 1972, Rosch Heider and Olivier 1972), they found no evidence that the supposedly universal or 'prototypical' colour categories named in English are either learned or remembered more easily than the best examples of the participants' own linguistic categories. A three-year, longitudinal study of colour term acquisition among young children learning to speak either English or Himba also found no learning advantage for English BCTs (Roberson et al. 2004). While these results don't conclusively establish that colour categorization is generally language-dependent, they do pose a challenge to the view that the process of BCT acquisition is guided by a pre-linguistic system that groups fine-grained shades into a universal set of coarse-grained categories.

Critics of linguistic relativism have put forward two main objections. The first has to do with patterns in colour-naming across different languages. Linguistic relativism is frequently taken to imply that colour naming is 'largely a matter of arbitrary linguistic convention' (Kay and Regier 2006: 52). But, if so, then lexical colour categorization systems could be expected to vary freely from one language to another. This prediction, however, is at odds with evidence for recurrent 
motifs in colour-naming practices discussed in the last section (Regier et al. 2005; Lindsey and Brown 2006, 2009).

Linguistic relativists have two ways of responding to this objection. The first is that the objection targets a straw man: colour-naming practices, according to the version of linguistic relativism advanced by Roberson and her colleagues, aren't arbitrary:

Even if there are genuine similarities between certain color systems, there are obvious cultural factors that could explain at least some of these similarities. Similar cultural needs, such as evolutionary pressure for successful frugivory, could also cause some category divisions to be more likely than others. Cultural contact between speakers of different languages has also clearly increased the similarity of the color categorization systems that these languages employ; for example, the term burou can be traced from German to Herero and subsequently to Himba.

(Robertson 2012: 42)

In addition to appealing to common cultural and environmental factors, linguistic relativists can also appeal to common categorization principles. Indeed, as pointed out at the end of the last section, recent universalist models have explored the hypothesis that cross-cultural patterns in colour categorization result from application of the informativeness constraint to an irregularly shaped colour space. Systems containing the same number of categories that conform to the informativeness constraint will partition colour space in similar, 'well-formed' ways (Regier et al. 2015).

Whereas the first objection had to do with evidence for convergence in colour-naming across different languages, the second objection has to do with evidence for intersubjective divergence in colour-naming within languages. Webster and Kay (2005) write:

[A] prominent property of actual color-naming data is the pronounced variation among speakers of the same language. ... For example, the wavelengths that individuals select for unique green within a linguistically homogeneous group span a range of more than $80 \mathrm{~nm}$; these variations are in fact so large that the same wavelength might be chosen as unique green by one observer and unique yellow or blue by another (Kuehni 2004). ... Mean foci across languages vary much less than individual foci within languages. This suggests that a common language imposes only a weak constraint, and a difference in language produces relatively little divergence.

(512; for a similar assessment, see Lindsey and Brown 2014: 524)

Two lines of response are open to the linguistic relativist. First, the surprising amount of withinlanguage variability in colour-naming is a problem for all theories of colour categorization and not just for linguistic relativism. (And, as pointed out in the last section, intersubjective differences in colour perception present a special challenge to theories that base colour-naming practices on panhuman universals of colour experience.) Second, intersubjective variation in colour processing may be smoothed over by linguistic charity: minor differences in colour naming may often be disregarded as irrelevant to speakers' communicative purposes (Jameson 2005b: 315). In this connection, it is important to investigate just how much intersubjective agreement in the use of a set of BCTs is actually required for effective communication within a group of speakers and, so, for the diachronic stabilization of a colour lexicon (Levinson 1997). To answer this question, it is necessary to know, among other things, how often fine-grained variations in colour appearance need to be communicated to ensure successful performance of individual and 
multi-agent tasks. Objects belonging to a certain artifactual or natural kind, for example, may vary quite a bit in colour appearance across subjects, but much of that variation may not affect how agents interact with or communicate about the kind.

Strong linguistic relativism goes beyond moderate linguistic relativism in maintaining that colour perception is categorical: learning to use a set of BCTs causes shades that fall within the boundaries of a named category to appear phenomenally more similar to one another in appearance and shades that fall on opposite sides of a category boundary to appear phenomenally less similar (LR4). In the next section, we will see that there is a substantial amount of evidence against this claim.

\section{Is colour perception categorical?}

A categorical perception $(\mathrm{CP})$ effect occurs

when (1) a set of stimuli ranging along a physical continuum is given one label on one side of a category boundary and another label on the other side and (2) the subject can discriminate smaller physical differences between pairs of stimuli that straddle that boundary than between pairs that are entirely within one category or the other.

(Harnad 1987: 3)

The paradigm of $\mathrm{CP}$ is phoneme discrimination in language: sounds straddling a phonemic category boundary, e.g. the boundary $/ \mathrm{ra} /$ and $/ \mathrm{la} /$ in English, are more discriminable to speakers of a language in which those phonemes occur than are sounds separated by equal acoustic step sizes, but from within the same phonemic category (Liberman and Mattingly 1985; Kuhl 2004). If colour perception is similarly categorical (LR4), then acquiring a set of colour terms could cause shades that straddle a named colour category boundary to appear phenomenally less similar (and, thus, easier to discriminate) and cause shades that fall within the boundaries of a named category to appear phenomenally more similar.

Tarahumara is an indigenous language of northern Mexico in which a single BCT ('siydname') is used to name both blue and green. In a classic study conducted by Paul Kay and Willett Kempton (1984), Tarahumara and English speakers were shown triads of Munsell colour chips in which only two of the chips fell on the same side of the blue-green boundary (whether blue or green). They were then asked to select the chip least similar in appearance to the other two. They found that English speakers were much more likely to choose the chip that fell on the other side of the blue-green boundary, even when within-category discrimination distances, as measure by justice noticeable difference (JND) steps, were greater than cross-category discrimination distances. Judgements made by Tarahumara speakers, by contrast, did not show any distorting effect of language and reflected objective discrimination distances.

What is the proper explanation of this effect? In a recent discussion, Jesse Prinz suggests that the 'presence of a linguistic color boundary between blue and green makes it impossible for English-speakers to perceive color distances objectively' (2012: 187). In other words, an object's apparent fine-grained shade of colour can vary as a function of the meanings of the colour terms present in a speaker's language, as maintained by strong linguistic relativism (LR4). Kay and Kempton, however, explicitly rejected this conclusion: 'it cannot be the case', they write, 'that the vision of English speakers is distorted in some way by the language they speak, because the discrimination distances that the Tarahumara faithfully reproduce on the subjective triads task were established on speakers of English' (1984: 72). Instead, they proposed that English speakers 
were relying on an unconscious, post-perceptual 'name strategy', when making their selections:

... faced with this situation the English-speaking subject reasons unconsciously as follows: 'It's hard to decide here which one looks the most different. Are there any other kinds of clues I might use? Aha! $A$ and $B$ are both CALLED green while $C$ is CALLED blue. That solves my problem; I'll pick $C$ as most different.' Of course this cognitive strategy, which we will call the 'name strategy', is not available to the Tarahumara speaker precisely because he or she doesn't have ready lexical labels for the concepts green and blue.

(Kay and Kempton 1984: 72)

To test the name strategy theory, Kay and Kempton conducted a second experiment using the same colour triads. In each trial, three chips were presented in a box with a sliding top that enabled subjects to compare only two chips at a time. The three chips were always arranged by hue, so that the middle chip was intermediate in hue between its flankers. Here is a description of their method:

Experimenter exposes pair $(A, B)$. 'You can see that this chip (points to $A$ ) is greener than this chip (points to $B$ ).' (All subjects readily agreed.) Experimenter slides cover so that $A$ is covered and $C$ exposed along with $B$; that is, the pair $(B, C)$ is now exposed, 'You can see that this chip (points to $C$ ) is bluer than this chip (points to $B$ ).' (Again all subjects agreed without problems.) 'Now', experimenter hands stimuli to subject, 'you may slide the cover back and forth as often as you like. I'd like you to tell me which is bigger: the difference in greenness between the two chips on the left or the difference in blueness between the two chips on the right.'

... The subject cannot reasonably ask himself (herself) whether chip $B$ is called green or blue because he (she) has already in effect both called it green and called it blue in agreeing to compare $B$ in greenness to $A$ and in blueness to $C$. It is thus irrelevant to this task whether chip $B$ would be called green or blue in another, neutral context.

(Kay and Kempton 1984: 73)

Under these conditions, English and Tarahumara speakers discriminated colours identically: 'Subjective similarity judgments follow discrimination distance and reflect no influence from lexical category boundaries' (1984: 73). This result suggests, contrary to strong linguistic relativism, that colour categories in language can exert a distorting influence on colour similarity judgements without having any effect on the way the colours themselves phenomenally appear. In other words, it suggests that colour CP effects are effects of colour language on post-perceptual decision-making or other cognitive processes and do not result from a 'distortion' of colour appearances near category boundaries. If this is right, then so-called colour categorical perception, as Roberson et al. (2009) put it, 'is categorical but not perceptual, and should rather be referred to simply as a category effect' (487, emphasis added). In the remainder of this section, I adopt this terminological recommendation.

Three additional sources of empirical evidence furnish support for the name strategy theory. First, subsequent studies have confirmed that language-relative colour category effects (CCEs) disappear with verbal interference (Roberson and Davidoff 2000; Gilbert et al. 2006; Winawer et al. 2007). Winawer and co-authors (2007), for example, looked for CCEs in speakers of Russian, who, unlike speakers of English, use distinct terms for dark blue (siniy) and light blue 
(goluboy). Subjects were shown three coloured squares arranged in a triad and were asked to judge which of the two squares on the bottom was identical in colour to the square on top. Winawer and co-authors found that Russian speakers' judgements were faster when the shades of the squares on the bottom straddled the siniy-goluboy boundary, than when they were from within the same category. English speakers did not show the same cross-category advantage. Consistent with Kempton and Kay's name strategy theory, CCEs in Russian speakers' discrimination performance disappeared when they performed a simultaneous verbal interference task.

This finding supports a 'dual code' model of the involvement of language in colour discrimination tasks (Roberson et al. 2008; Winawer and Witthoft 2012). Jon Winawer and Nathan Witthoft (2012) write in a passage worth quoting at length:

If a category effect goes away when labels become unavailable or not useful, then it is unlikely that the effect is due to color terms affecting early perceptual processes. While such an account is logically possible, it would require color appearance to be altered only during those moments when one is accessing the labels. A more parsimonious explanation is that the decision process is affected by language. Verbal labels may be used to help keep track of the various stimuli in an experiment, either over a memory delay or when comparing stimuli spread over space. If, on a particular trial, all the stimuli come from the same verbal category (e.g., they are all blue), then labels are unlikely to help accomplish the task (and might even hinder performance). In contrast, if stimuli in a trial can easily be assigned different labels (e.g., one blue and one green), then access to the labels may facilitate memory or the comparison process. If a verbal dual task interferes with the ability to label stimuli, even implicitly, then this may eliminate one strategy or source of information for accomplishing the task, and hence may change performance. Thus, verbal interference effects are more likely to reflect a role of color terms on decisions, strategy, and memory, rather than perception.

A second source of evidence for the name strategy explanation comes from studies that have found CCEs to be significantly stronger on the right side of the visual field (RVF) than on the left (LVF) (Gilbert et al. 2006; Roberson et al. 2008; Roberson and Pak 2009; for a review, see Regier and Kay 2009). This is relevant because stimuli presented in the RVF project to the left hemisphere of the brain, which is typically dominant for language. ${ }^{7}$

A final source of evidence for the name strategy theory comes from experiments that have investigated JND thresholds among speakers of languages with different lexicalized colour categories. If language 'stretches' perceptual distances at boundaries between colour categories (LR4), then discrimination thresholds should be lower at category boundaries, that is, shades near category boundaries should be more finely discriminated, than near category centres. ${ }^{8}$ Contrary to predictions based on LR4, Roberson et al. (2009) found that colour discrimination is neither enhanced for English speakers at the boundary between blue and green boundary, nor for Korean speakers at the boundaries between categories that are named in Korean, but not in English. 'In the case of color', they suggest, 'humans may already have hyper-acuity (Churchland \& Sejnowski 1994), so that no further "tuning" occurs with category learning' (486). ${ }^{9}$

\section{Conclusion}

In concluding this chapter, it may be helpful to review some main points of convergence between universalists and linguistic relativists about colour categorization. To begin with, there 
is general agreement on the existence of interesting patterns in colour-naming across speakers of different languages. Moreover, it is now widely accepted that these patterns are not supported by the distinctive appearance or perceptual salience of the Hering primaries (4UH plus black and white). Second, prominent universalists now accept that colour category effects (CCEs) are language relative. Kay and Regier (2007), for example, agree that 'there is ample evidence that differences in color category boundaries between languages may influence color memory, learning or discrimination' (294). In other words, CCEs don't indicate the existence of pre-linguistic colour concepts that constrain the construction of colour categorization systems across speakers of different languages. Finally, contemporary linguistic relativists have distanced themselves from the Whorfian view that colour language can modulate the phenomenology of colour experience: CCEs reflect the influence of colour terms on memory and decision-making rather than on the way fine-grained shades of colour visually appear.

\section{Notes}

1 The number of perceptually distinct surface colours actually present in natural scenes, however, is probably much smaller (Marín-Franch and Foster 2010).

2 A colour term is said to be basic, when among other things it is monolexemic, e.g. 'blue' vs. 'sky blue' or 'dark blue', applied to different types of objects, and used by most speakers of the language in which it occurs (Berlin and Kay 1969). For discussion, see the chapter by Dedrick in this volume.

3 Also see the target articles by Saunders and van Brakel (1997) and Steels and Belpaeme (2005) in Behavioral and Brain Sciences and their invited commentaries for valuable discussions.

4 It should be emphasized, however, that Byrne and Hilbert (1997) reject the claim that the perceptual salience of the Hering primaries is by itself explanatory of colour-naming practices.

5 For discussions of cognitive or 'extracommunicative' theories of language, see Clark (1998) and Carruthers (2002).

6 The perceptual salience theory, by contrast, maintains that colour categorization systems in all languages are based on a universal set of chromatic landmarks-black, white, UR, UY, UG, and $\mathrm{UB}$ - and, accordingly, that hue is universally the most heavily weighted dimension of variation in colour appearance.

7 It should be emphasized that the existence of CCEs in the RVF is not uncontroversial. Brown et al. (2011) found no CCE on visual search reaction times involving stimuli at the blue-green boundary presented in either visual field. 'Taken as a whole', they write, 'the results and analyses suggested that the overall shape of the [reaction time] data sets was controlled entirely by visual signals that arise in the cones and are combined in a color-opponent fashion in the earliest stages of visual processing' (2).

8 Winawer and Witthoft (2012: 6) write:

Threshold discrimination experiments are among the least ambiguous experiments in psychology. If an observer can discriminate two stimuli, then we can be certain that the observer's perceptual system has encoded the two stimuli differently. If the stimuli are indistinguishable (below threshold), then information distinguishing the stimuli was either not encoded or was lost in subsequent processing. If discrimination thresholds were altered by the color terms in one's language, this would provide the most direct evidence that color terms affect perception of colors.

9 Regier and Kay (2009) review evidence that prior to language acquisition colour categories may be represented in the right hemisphere and cause CCEs in the LVF of young infants (Bornstein et al. 1976; Franklin et al. 2005; Franklin et al. 2008). For present purposes, it is important to emphasize that even if pre-linguistic infants do exhibit CCEs (but for a sceptical assessment, see Roberson and Hanley (2009)), the relevant effects don't appear to facilitate colour term acquisition or have any other effects on later colour cognition. The psychologist Marc Bornstein observes: 'An otherwise reasonable surmise from the fact that hue characterization precedes color naming developmentally would be that, in this one realm at least, linguistic identification simply overlays perceptual cognitive organization and thereby facilitates semantic development. Paradoxically, it does not' (1985: 74). After language is learned, right hemisphere categories appear to be 'permanently erased' (Regier and Kay 2009: 441). For critical discussion of 'Bornstein's paradox', see Dedrick (2014b). 


\section{Categorization and categorical perception}

\section{References}

Berlin, B., and Kay, P. (1969). Basic Color Terms: Their Universality and Evolution. Berkeley, CA: University of California Press.

Bornstein, M. H. (1985). On the development of color naming in young children: Data and theory. Brain and Language, 26, 72-93.

Bornstein, M. H., Kessen, W., and Weiskopf, S. (1976). Color vision and hue categorization in young human infants. Journal of Experimental Psychology: Human Perception and Performance, 2, 115-29.

Bosten, J. M., and Boehm, A. E. (2014). Empirical evidence for unique hues? Journal of the Optical Society of America, 31(4), A385-93.

Bosten, J. M., and Lawrance-Owen, A. J. (2014). No difference in variability of unique hue selections and binary hue selections. Journal of the Optical Society of America, 31, A357-64.

Boynton, R. (1997). Insights gained from naming the OSA colors. In C. L. Hardin (Ed.), Color Categories in Thought and Language. Cambridge: Cambridge University Press, pp. 135-50.

Broackes, J. (2011). Where do the unique hues come from? Review of Philosophy and Psychology, 2, 601-8.

Brown, A. M., Lindsey, D. T., and Guckes, K. M. (2011). Color names, color categories, and color-cued visual search: Sometimes, color perception is not categorical. Journal of Vision, 11, 2-21.

Byrne, A., and Hilbert, D. R. (2003). Color realism and color science. Behavioral and Brain Sciences, 26(1), 3-21.

Carruthers, P. (2002). The cognitive functions of language. Behavioral and Brain Sciences, 25, 657-726.

Churchland, P. S., and Sejnowski, T. J. (1994). The Computational Brain. Cambridge, MA: MIT Press.

Chomsky, Noam. (1995). The Minimalist Program. Cambridge, MA: MIT Press.

Clark, A. (1998). Magic words. In P. Carruthers and J. Boucher (Eds.), Language and Thought: Interdisciplinary Themes. Cambridge: Cambridge University Press, pp. 162-83.

Davidoff, J. (2001). Language and perceptual categorisation. Trends in Cognitive Sciences, 5(9), 382-7.

Davidoff, J., and Roberson, D. (2004). Preserved thematic and impaired taxonomic categorisation: A case study. Language and Cognitive Processes, 19(1), 137-74.

Davidoff, J., Davies, I., and Roberson, D. (1999). Colour categories of a stone-age tribe. Nature, 398, 203-4.

Dedrick, D. (1997). Colour categorization and the space between perception and language. Behavioral and Brain Sciences, 20, 187-8.

Dedrick, D. (1998). Naming the Rainbow: Colour Language, Colour Science, and Culture. Dordrecht: Kluwer.

Dedrick, D. (2014a). Colour language, thought, and culture. In F. Sharifiian (Ed.), Routledge Handbook to Mind, Language and Culture. New York: Routledge, pp. 270-93.

Dedrick, D. (2014b). Bornstein's paradox (redux). In W. Anderson, C. P. Biggam, C. Hough, and C. Kay (Eds.), Colour Studies: A Broad Spectrum. Amsterdam: John Benjamins, pp. 181-99.

Franklin, A., Clifford, A., Williamson, E., and Davies, I. R. L. (2005). Color term knowledge does not affect categorical perception of color in toddlers. Journal of Experimental Child Psychology, 90, 114-41.

Franklin, A., Drivonikou, G. V., Bevis, L., Davies, I. R. L., Kay, P., and Regier, T. (2008). Categorical perception of color is lateralized to the right hemisphere in infants, but to the left hemisphere in adults. Proceedings of the National Academy of Sciences of the United States of America, 105, 3221-5.

Garner, W. R. (1974). The Processing of Information and Structure. Hillsdale, NJ: Erlbaum.

Gilbert, A. L., Regier, T., Kay, P., and Ivry, R. B. (2006). Whorf hypothesis is supported in the right visual field but not the left. Proceedings of the National Academy of Sciences of the United States of America, 103, 489-94.

Hardin, C. L. (1988). Color for Philosophers: Unweaving the Rainbow. Indianapolis, IN: Hackett.

Hardin, C. L. (2005). Explaining basic color categories. Cross-Cultural Research: The Journal of Comparative Social Science, 39, 72-87.

Harnad, S. (1987). Psychophysical and cognitive aspects of categorical perception: A critical overview. In S. Harnad (Ed.), Categorical Perception: The Groundwork of Cognition. Cambridge: Cambridge University Press, pp. 1-28.

Hering, E. (1878/1964). Grundzüge der Lehre vom Lichtsinn (Outlines of a Theory of the Light Sense). L. M. Hurvich and D. Jameson (trans.). Cambridge, MA: Harvard University Press.

Jameson, K. A. (2005a). Why GRUE? An interpoint distance model analysis of composite color categories. Cross-Cultural Research, 39, 159-204.

Jameson, K. A. (2005b). Culture and cognition: What is universal about the representation of color experience? The Journal of Cognition \& Culture, 5(3-4), 293-347. 


\section{Robert Briscoe}

Jameson, K. A., and D'Andrade, R. G. (1997). It's not really red, green, yellow, blue: An inquiry into cognitive color space. In C. L. Hardin and L. Maffi (Eds.), Color Categories in Thought and Language. Cambridge: Cambridge University Press, pp. 295-319.

Jameson, K. A., and Komarova, N. L. (2009a). Evolutionary models of color categorization. I. Journal of the Optical Society of America, A, 26, 1414-23.

Jameson, K. A., and Komarova, N. L. (2009b). Evolutionary models of categorization. II. Journal of the Optical Society of America, A, 26, 1424-36.

Kay, P., and Kempton, W. (1984). What is the Sapir-Whorf hypothesis? American Anthropologist, 86, 65-78.

Kay, P., and Maffi, L. (1999). Color appearance and the emergence and evolution of basic color lexicons. American Anthropologist, 101, 743-60.

Kay, P., and McDaniel, C. (1978). The linguistic significance of the meanings of basic color terms. Language, 54, 610-46.

Kay, P., amd Regier, T. (2006). Language, thought and color: Recent developments. Trends in Cognitive Sciences, $10(2), 51-4$.

Kay, P., and Regier, T. (2007). Color naming universals: The case of Berinmo. Cognition, 102, $289-98$.

Kay, P., Berlin, B., Maffi, L., Merrifeld, W. R., and Cook, R. (2009). The World Color Survey. Stanford, CA: Center for the Study of Language and Information.

Kuehni, R. G. (2004). Variability in unique hue selection: A surprising phenomenon. Color Research and Application, 29, 158-62.

Kuehni, R. G. (2005a). Unique hue stimulus choice: A constraint on hue category formation. Journal of Cognition and Culture, 5(3-4), 387-408.

Kuehni, R. G. (2005b). Focal color variability and unique hue stimulus variability. Journal of Cognition and Culture, 5(3-4), 409-26.

Kuehni, R. G. (2013). Color: An Introduction to Practice and Principles, 3rd ed. Hoboken, NJ: John Wiley $\&$ Sons.

Kuhl, P. K. (2004). Early language acquisition: Cracking the speech code. Nature Reviews Neuroscience, 5(11), 831-43.

Levinson, S. C. (1997). Yêlî dyne and the theory of basic color terms. Paper presented in a seminar at the Max Plank Institute for Psycholinguistics, June 1997.

Liberman, A. M., and Mattingly, I. G. (1985). The motor theory of speech perception revised. Cognition, $2,1-36$.

Lindsey, D. T., and Brown, A. M. (2006). Universality of color names. Proceedings of the National Academy of Sciences of the United States of America, 103, 16608-13.

Lindsey, D. T., and Brown, A. M. (2009). World Color Survey color naming reveals universal motifs and their within-language diversity. Proceedings of the National Academy of Sciences of the United States of America, 106, 19785-90.

Lindsey, D. T., and Brown, A. M. (2014). Color appearance, language, and neural coding. In J. S. Werner and L. M. Chalupa (Eds.), The New Visual Neurosciences. Cambridge, MA: MIT Press, pp. 511-31.

Linhares, J. M. M., Pinto, P. D., and Nascimento, S. M. C. (2008). The number of discernible colors in natural scenes. Journal of the Optical Society of America A, Optics, Image Science, and Vision, 25, 2918-24.

Lupyan, G. (2009). Extracommunicative functions of language: Verbal interference causes selective categorization impairments. Psychonomic Bulletin \& Review, 16(4), 711-18.

Lupyan, G., and Mirman, D. (2013). Linking language and categorization: Evidence from aphasia. Cortex, 49(5), 1187-94.

Malkoc, G., Kay, P., and Webster, M. A. (2002). Individual differences in unique and binary hues. Journal of Vision, 2: 32a.

Malkoc, G., Kay, P., and Webster, M. A. (2005). Variations in normal color vision. IV. Binary hues and hue scaling. Journal of the Optical Society of America A, 22, 2154-68.

Marín-Franch, I., and Foster, D. (2010). Number of perceptually distinct surface colors in natural scenes. Journal of Vision, 10(9), 1-7.

Pointer, M. R., and Attridge, G. G. (1998). The number of discernible colours. Color Research E Application, 23(1), 52-4.

Prinz, J. (2012). Beyond Human Nature. New York: W. W. Norton \& Company.

Quine, W. V. O (1973). The Roots of Reference. La Salle: Open Court.

Ray, V. (1953). Human color perception and behavioral response. Transactions of the New York Academy of Sciences, 2(16), 98-105. 


\section{Categorization and categorical perception}

Regier, T., and Kay, P. (2009). Language, thought, and color: Whorf was half right. Trends in Cognitive Sciences, 13, 439-46.

Regier, T., Kay, P., and Cook, R. S. (2005). Focal colors are universal after all. PNAS, 102, 8386-91.

Regier, T., Kay, P., and Khetarpal, N. (2007). Color naming reflects optimal partitions of color space. Proceedings of the National Academy of Sciences of the United States of America, 104, 1436-41.

Regier, T., Kay, P., Gilbert, A., and Ivry, R. (2010). Language and thought: Which side are you on, anyway? In B. Malt and P. Wolff (Eds.), Words and the Mind: How Words Capture Human Experience. New York: Oxford University Press, pp. 165-82.

Regier, T., Kemp, C., and Kay, P. (2015). Word meanings across languages support efficient communication. In B. MacWhinney and W. O'Grady (Eds.), The Handbook of Language Emergence. Hoboken, NJ: Wiley-Blackwell, pp. 237-63.

Roberson, D. (2005). Color categories are culturally diverse in cognition as well as in language. CrossCultural Research, 39(1), 56-71.

Roberson, D. (2012). Culture, categories and color-Do we see the world through t(a)inted lenses? In M. Gelfand, C. Chiu, and Y. Hong (Eds.), Advances in Culture and Psychology (Vol. 2). New York: Oxford University Press, pp. 3-52.

Roberson, D., and Davidoff, J. (2000). The categorical perception of colors and facial expressions: The effect of verbal interference. Memory and Cognition, 28, 977-86.

Roberson, D., and Hanley, J. R. (2009). Relatively speaking: What is the relationship between language and thought in the color domain? Glimpse, 2(3), 68-77.

Roberson, D., and Pak, H. S. (2009). Categorical perception of color is restricted to the right visual field in Korean speakers who maintain central fixation. Journal of Cognitive Science, 10, 41-51.

Roberson, D., Davidoff, J., and Braisby, N. (1999). Similarity and categorization: Neuropsychological evidence for a dissociation in explicit categorization tasks. Cognition, 71, 1-42.

Roberson, D., Davies, I. R. L., and Davidoff, J. (2000). Colour categories are not universal: Replications and new evidence from a stone-age culture. Journal of Experimental Psychology: General, 129, 369-98.

Roberson, D., Davidoff, J., Davies, I. R. L., and Shapiro, L. R. (2004). The development of color categories in two languages: A longitudinal study. Journal of Experimental Psychology: General, 133, 554-71.

Roberson, D., Davidoff, J., Davies, I. R. L., and Shapiro, L. (2005a). Colour categories in Himba: Evidence for the cultural relativity hypothesis. Cognitive Psychology, 50, 378-411.

Roberson, D., Davies, I. R. L., Corbett, G., and Vandervyver, M. (2005b). Free-sorting of colors across cultures: Are there universal grounds for grouping? Journal of Cognition and Culture, 5, 349-86.

Roberson, D., Hanley, J. R., and Pak, H. (2009). Thresholds for color discrimination in English and Korean speakers. Cognition, 112, 482-7.

Roberson, D., Pak, H. S., and Hanley, J. R. (2008). Categorical perception of colour in the left and right visual field is verbally mediated: Evidence from Korea. Cognition, 107, 752-62.

Rosch, E. (1973). Natural categories. Cognitive Psychology, 4, 328-50.

Rosch Heider, E. (1972). Universals in color naming and memory. Journal of Experimental Psychology, 93, 10-20.

Rosch Heider, E., and Olivier, D. (1972). Universals in color naming and memory. Journal of Experimental Psychology, 93, 10-20.

Saunders, B. A. C., and van Brakel, J. (1997). Are there non-trivial constraints on color categorization? Behavioral and Brain Sciences, 20, 167-78.

Shepard, R. N. (1992). The perceptual organization of colors: An adaptation to regularities of the terrestrial world? In J. H. Barkow, L. Cosmides, and J. Tooby (Eds.), The Adapted Mind: Evolutionary Psychology and the Generation of Culture. New York: Oxford University Press, pp. 495-532.

Smallman, H., and Boynton, R. (1990). Segregation of basic colours in an information display. Journal of the Optical Society of America A, 7(10), 1985-94.

Smallman, H., and Boynton, R. (1993). On the usefulness of color coding in an information display. Displays, $14,158-65$.

Steels, L., and Belpaeme, T. (2005). Coordinating perceptually grounded categories through language: A case study for colour. Behavioral and Brain Sciences, 28, 469-89.

Sternheim, C. E., and Boynton, R. M. (1966). Uniqueness of perceived hues investigated with a continuous judgmental technique. Journal of Experimental Psychology, 72(5), 770-6.

Webster, M. A., and Kay, P. (2005). Variations in color naming within and across populations. Behavioral and Brain Sciences, 28, 512-13. 


\section{Robert Briscoe}

Whorf, B. L. (1956). Language, Thought, and Reality: Selected Writings of Benjamin Lee Whorf. Cambridge, MA: Technology Press of Massachusetts Institute of Technology.

Winawer, J., and Witthoft, N. (2012). Effects of color terms on color perception and cognition. In Encyclopedia of Color Science and Technology. Springer: New York, pp. 1-8. doi:10.1007/Springer Reference_300496.

Winawer, J., Witthoft, N., Frank, M. C., Wu, L., Wade, A. R., and Boroditsky, L. (2007). Russian blues reveal effects of language on color discrimination. Proceedings of the National Academy of Sciences of the United States of America, 104, 7780-5.

Yendrikhovskij, S. N. (2001). Computing color categories from statistics of natural images. Journal of Imaging Science and Technology, 45, 409-17. 